\title{
Predicting the Best Team Players of Pakistan Super League using Machine Learning Algorithms
}

\author{
Jawaria Ashraf \\ MS Scholar \\ Institute of Information and \\ Communication Technology, \\ Mehran UET, Jamshoro, Pakistan
}

\author{
Sania Bhatti, PhD \\ Associate Professor \\ Department of Software \\ Engineering, Mehran UET, \\ Jamshoro, Pakistan
}

\author{
Shahnawaz Talpur, PhD \\ Associate Professor \\ Institute of Information and \\ Communication Technology, \\ Mehran UET, Jamshoro, Pakistan
}

\begin{abstract}
Owing to short and fast paced play, T20 is the adored format of cricket sport. In T20 cricket, Pakistan super league (PSL) is one of the most famous professional leagues founded to strengthen Pakistan cricket by scrutinizing the young talent. However, the selection of the best players for PSL teams is a very critical phase which certainly affects the final results of the play. To avoid biasness caused by the human nature in selection process, this study aims to select and rank the team of top fifteen players based on their batting and bowling performance in previous five seasons of PSL using Machine learning approach. For this purpose, Support vector machine (SVM), Random forest, Naive Bayes, Linear regression and K-nearest neighbor (classification) techniques have been employed for the development of predictive model from individual batting and bowling features sets. Based on comparison of applied techniques, the evaluated results have been plotted in term of accuracy, precision, recall and "flscore". For the selection of both batsman (in term of runs scored) and bowlers (in term of wickets taken), Random Forest performed well by yielding an accuracy of $100 \%$. Findings of this research also ascertain that batting performance leads over bowling performance.
\end{abstract}

\section{Keywords}

Pakistan Super League, Batting, Bowling, Machine Learning, Prediction, Classification, Ranking

\section{INTRODUCTION}

Cricket is renowned, simple and entertainment sport around the globe especially in Asian countries. In the southeast England, cricket sport was initiated in the 16th century and became an international team play of the world. Cricket is played between two teams each consists of 11 players. Three main formats of cricket including one day international, test and T20s are played internationally [1]. T20 is the most popular format among fans due to its shorter and fast paced play. Therefore, this research focuses on T20s format due to its popularity at international as well as domestic level. In T20 cricket, both squads are mainly concerned with a maximum of 20 overs in each inning. There are a number of T20 domestic leagues played around the globe. Pakistan super league is the one of the most famous T20 cricket domestic leagues which is admired due to its competitiveness in term of batting and bowling. PSL was established in 2015 with 5 cricket teams including Peshawar Zalmi, Quetta Gladiators, Islamabad United, Lahore Qalandars, Karachi Kings and Multan Sultan was introduced in season 3 as 6th team. PSL usually starts in February, in which every team play 2 matches against each team. Top 4 best performing teams are qualified for semifinal. Out of 4 teams, only two teams qualify for final match and terminating the tournament by winning PSL Cup. Each PSL team selects highly qualified international players from 11 different countries of the world. Selection committee selects the players including wicket keeper, batsmen and bowlers. Each type of player has its specialized skills like batsman is enough competent to bat at various locations, angles and different types of bowlers including spin bowler, fast bowler and medium fast bowler. People of various era and surroundings are extreme buffs of cricket owing to well performance of cricket team within recent bygone. Though, several pregame misperceptions ascend regarding team combo as which batsman and bowler should be nominated or dropped and which batting player have to play in which position for forthcoming match because it's a sport of uncertainty to predict the final results. Several ordinary aspects, matches' instructions, players' talent, their coordination and practice arrangements are very significant to analyze the performance metrics of final outcome of the match which is very helpful for team managers, instructors and speculators too [2]. Machine learning approaches are used by emerging classification methods based on significant features which directly impact on the final outcome of the match as weather, position of the players, location, home team, toss decision to estimate match outcome [3,4]. The model is established to calculate the proficiency of the final result of the match using method contains training the data based on historical matches [5]. Usually, efficiency of machine learning approach is estimated in performance metric as prediction accuracy [6]. From the previous record of past 5 PSL seasons, it has been observed that selection of the players highly affects the final results of the match and it is also difficult to take a decision whether batting capabilities lead over bowling capabilities or not. Hence, to improve the team winning chance of tournament, the team players must be composed and categorized. Moreover, winning prediction also rely on features including toss winning, home ground, pitch , batting and bowling classification in term of run rate, batting average, bowling average, wickets taken and so on. So, one of the foremost techniques applied in selection of the top players research is machine learning which plays an important role in evaluation parameters of the final results of the match. Subsequently final outcome of the matches is evaluated using machine learning classification methods with several input factors based on previous matches played.

In this research, various machine learning approaches are employed to determine the best models which can evaluate the final result of the match with high performance metrics. The research interrogation we try to response is:

- For cricket matches allied to PSL-T20, which machine learning technique procure best probabilistic model and which classification 
approaches are accurate in term of evaluation parameters including "accuracy", "precision", "recall" and "fl-score"?

The riposte of research query is that various machine learning techniques are empirically observed from previous studies containing decision tree, random forest, SVM and naive Bayes [7, 8] for best model which can evaluate the final result of the match with high accuracy. In this research we used previous 5 years of PSL data which is collected from PSLT20 tournaments. Further description on data and experimental outcomes are deliberated in section 3 and section 4 correspondingly. The aim of this research is to identify the features influencing the performance which have direct impact on the final outcome of the match in cricket field. This research fascinated on producing an intelligible and simplified model which is effective to analyze the match result based on batting and bowling performances using machine learning prediction techniques for upcoming match series. Main reason behind the prediction of the match result is to enhance team capabilities and to uplift the team winning chances of tournament. Significance of team winning is beneficial for television indentures, enthusiast stock market, membership and maintenance, financial and media sponsorships, enterprises and stadium arrangements. This research can help the cricket management and researchers interested in cricket data prediction.

To have clear understanding, this paper is distributed in five different sections. After introduction, literature review is presented in section 2. Section 3 describes the methodology of the paper. Section 4 discusses classification results. Finally, section 5 of the paper concluded the findings with future research directions.

\section{LITERATURE REVIEW}

In this section, previous research to be acquainted with comprehensive study in cricket field is completed. Investigations fanatical to this field are deliberated in detail here. Many researchers have done prediction of cricket match result with several essential features like toss decision, home ground, day /night, performance of players, bowling and batting using machine learning prediction techniques classification via regression. The research mechanism is interconnected to several cricket match problems which are enlightened below.

Jayalath, K.P. [1] described classification, regression tree and logistic regression algorithms to predict ODI cricket match impact. In this research, the authors have examined the significance of home field advantage for several clubs including Pakistan, South Africa, India and New Zealand with respect to challenger's field location. In the main output result amongst all the team, by using CART and Logistic regression the South Africa has maximum winning possibility about $72 \%$ in home team advantage. Kumashkapadia et al. [3] examined machine learning algorithms to predict the IPL-T20 match. Authors have used filter-based model to identify significant features of the dataset and approved four Machine learning techniques comprising K-Nearest Neighbor, Naïve Bayes, Model Trees and Random Forest to predict the match outcomes from these distinctive features. Experimental result showed that Naïve Bayes algorithm generated better results for home team advantage feature to improve predictive outcomes. Munir, F., et al. [4] reciprocated in-play and preplay data to estimate optimal output. They evaluated T20 format of international matches and IPL cricket match data as training dataset. In deep study, they divided the data on the base of various features like batting first, location, and home team vs. opponent team etc. Decision tree algorithm gave the best results with the accuracy of $78 \%$ and $75 \%$ for first inning and second inning respectively. Kampakis et al. [5] applied gradient decision trees, naive Bayes and logistic regression etc. approaches on particular dataset to predict the match outcome. In this model naïve Bayes generate better result and gave high accuracy about $64 \%$ while gradient decision trees gave lowest accuracy in the comparative study. Ahmed, et al. [6] utilized machine learning models with features selection and splitting of data for pre-match prediction. Pakistan's cricket performance prediction was done by random forest method with $82 \%$ accuracy. Pre-competition scrutiny which they used was the major reason to avoid any intolerant view and unsafe reaction. AkhilNimmagadda et al. [9] introduced T20 cricket match prediction model while the match is in progress [4]. They used statistical techniques to get the best results for predictive system. Regression model and random forest classifier applied to predict outcome of both first and second innings which based on runs scored per over. They obtained winner of the match by using random forest technique. NeerajPathak and HardikWadhwa [10] worked on predicting the optimum result for ODI match based on many features including toss decision, innings, home ground, performance of team players etc. They have used new classification approaches like naïve bayes, SVM, and random forest $[3,8]$ to generate all possibilities of winning or losing of an ODI cricket match (2001-2015). Experimental results showed that the SVM approach performed better with $62 \%$ accuracy while other models gave $60 \%$ accurate result.MadanGopalJhanwar and VikramPudi[11] applied supervised learning approach to predict the outcome of ODI cricket. Firstly KNN, SVM, Random forests, Logistic regression and Decision trees techniques were applied [12] on 22 player's performance to calculate the final results of match. KNN performed better results than other classifier and also gave high accuracy about $71 \%$.Somaskandhan, P. [13] determined the important set of features which are highly affected on cricket match end results. They introduced twenty three various attributes to describe the facts of inning level and calculated them. In this research, various machine learning techniques are used but SVM is best approach to obtain optimal result with high accuracy.Thenmozhi, D et al. [14] elucidated several classification techniques to predict that the home team can win the match or not when the match is in progress. In the end result, the optimal output is obtained through Random Forest model because this technique provided highest accuracy for all of the team.

From the previous research, it is evident that most of studies have been conducted on machine learning methods for the prediction of final results of the match using different features and it is also identified that all researchers have used different numeral of attributes and machine learning methods in their paper. They are also directing different formats of cricket in their research. Some scholars have deliberated only features in their work while some scholars have evaluated which machine learning model will be accurate to predict the cricket match results. From above literature the low performance accuracy is achieved in study [10] which is $60 \%$ and highest performance accuracy is achieved in study [6] which is $82 \%$. In certain studies, insufficient parameters are used which may decrease the performance accuracy of evaluated output. Therefore, we have selected important features and topmost approach too which can improve the performance metrics as prediction accuracy with best results. However, a limited research has been directed on the effects of players' selection on the final 
outcomes of the match. Therefore, this study focuses on the prediction of the final results based on the selection of the players using different machine learning approaches before match. In this work, we used random forest, SVM, naive Bayes, KNN and linear regression methods because in literature these classifiers are ideal for such dataset.

\section{METHODOLOGY \& DATA DESCRIPTION}

\subsection{Methodology}

In this research, several machine learning approaches are evaluated to tackle the problem of predicting final result of PSL match series. Intellectual models are depicted to analyze the result of the match based on the influence of batting and bowling performances respectively to improve the winning chance of tournament. For this purpose, two predictive models representing the effect of batting capabilities and bowling capabilities of PSL players are formulated to select the top batsmen and bowlers correspondingly. To estimate the batting and bowling performance of top players of PSL using machine learning methods, the Scikit library in Anaconda as package and Jupyter notebook as IDE is used. Figure 1 signifies the methodology steps of this research to evaluate the final outcomes.

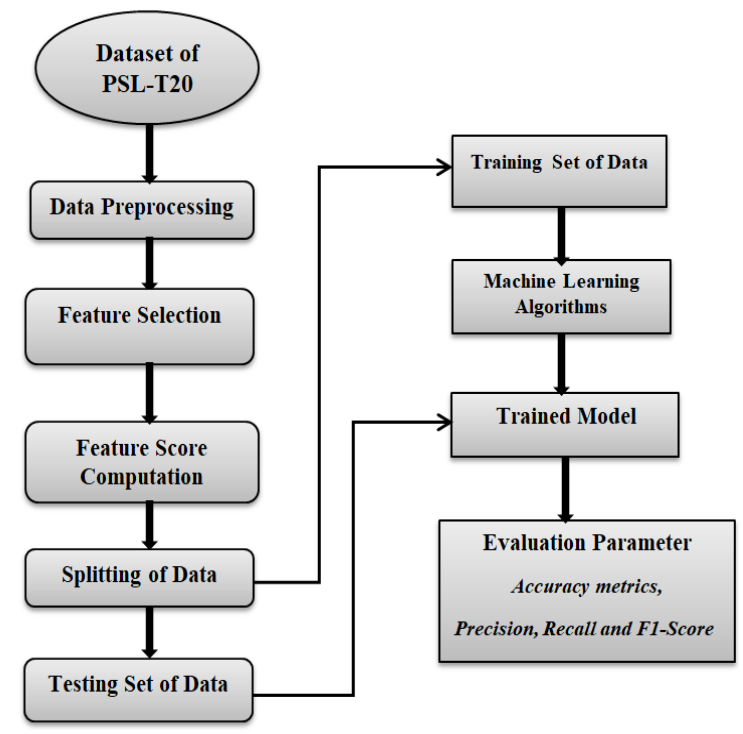

Fig 1: Proposed methodology

First, the input features of batting and bowling dataset are preprocessed by excluding insufficient parameters and choosing the key features which directly impact on training set's performance using feature selection method. Then extract the individual value of each feature using correlation matrix and compute the feature score. After feature computation, the dataset is split into various sizes of dualistic percentages (training-data and testing-data) to obtain accurate combination which provides high accuracy. Selection of top players is based on maximum runs made by batsmen and maximum wickets taken by bowlers respectively by means of different parameters. Then machine learning approaches are applied to develop classification models. These predictive models are finally compared with precision, accuracy, recall and "fl-score" to analyze the final results.

\subsection{Data Collection}

Previous five years of Pakistan super league (PSL-T20) data is apprehended to execute valuation scrutiny. So, this research uses the secondary statistics which is easily accessible on Cricinfo website. From [15] we extracted the required data for series of PSL season I-V (2016-2020). Data consists of several features as 16 factors and 526 instances for batsmen dataset and bowlers' dataset respectively. Then, we analyzed the data, selected the significant features and created a CSV file. As per PSL procedures only 5 separate teams take part in every single season of tournament. Though, one more team has been made in season 3 (2018) which is Multan Sultan. Owing to this, PSL dataset has 6 separate teams. Majority of PSL tournaments are played by Peshawar Zalmi, Quetta Gladiators, Islamabad United, Lahore Qalandars and Karachi Kings whereas Multan Sultan played only three PSL seasons. Figure 2 shows the dataset of batsmen and bowler's performance before preprocessing of the data.

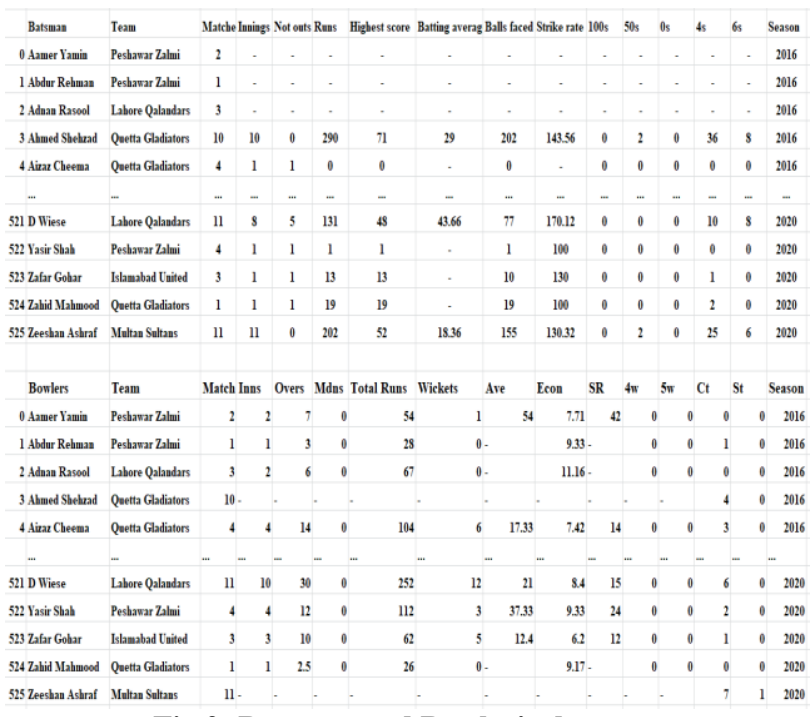

Fig 2: Batsmen and Bowler's dataset

\subsection{Feature Selection}

Data is preprocessed using Pandas library in Jupyter notebook to select the effective parameters from batsman and bowlers dataset to predict the final outcomes of the match which minimizes overfitting, improves performance accuracy and fast to train the model. To get rid of missing records in dataset, the independent variables are preprocessed as input dataset by excluding inadequate proceedings. PSL statistics with no prediction of match outcome was eradicated from machine learning classification models. Finally 13 features are chosen for batting performance and 9 features for bowling performance that acknowledged as accurate set of attributes using wrapper and filter based feature selection method. Several attributes influence the cricket match [16]. Moreover significant features which affect the cricket match based on batting and bowling proficiencies are used to rank the top players which are listed below in Table 1 and Table 2 respectively. 
Table 1. Batting attributes

\begin{tabular}{|l|l|}
\hline $\begin{array}{l}\text { Batting } \\
\text { features }\end{array}$ & Description \\
\hline Batsmen & $\begin{array}{l}\text { Name of the Batsmen who played in PSL-T20 } \\
\text { tournament. }\end{array}$ \\
\hline Runs & $\begin{array}{l}\text { Total runs recorded by batsman in PSL series. } \\
\text { Maximum record shows the best performance } \\
\text { of batsman. }\end{array}$ \\
\hline Matches & Total matches played by batsman in PSL series. \\
\hline $\begin{array}{l}\text { Highest } \\
\text { score }\end{array}$ & Highest score made by batsman in PSL series. \\
\hline $\begin{array}{l}\text { Batsman' } \\
\text { strike rate }\end{array}$ & $\begin{array}{l}\text { Total runs score recorded for every hundred } \\
\text { balls which faced by batsman voguish PSL } \\
\text { series. } \\
\text { Batting strike } \\
\text { runs*100)/balls faced by batsman }\end{array}$ \\
\hline $\begin{array}{l}\text { Batting } \\
\text { average }\end{array}$ & $\begin{array}{l}\text { Total runs scored by batsman per total intervals } \\
\text { in which he baptized as out in PSL series. } \\
\text { Batting Average = (Batsman runs/ number of } \\
\text { innings) }\end{array}$ \\
\hline Innings & $\begin{array}{l}\text { Total innings in which Batsman essentially } \\
\text { played in PSL series. }\end{array}$ \\
\hline Balls faced & $\begin{array}{l}\text { Sum of balls faced by batsman in PSL series } \\
\text { containing no balls. }\end{array}$ \\
\hline Not outs & Batsman who not outs in PSL series. \\
\hline $\begin{array}{l}\text { Centuries } \\
(100 s)\end{array}$ & $\begin{array}{l}\text { Total innings in which batsman recorded } \\
\text { hundred score in PSL series. }\end{array}$ \\
\hline Fifties (50s) & $\begin{array}{l}\text { Total innings in which batsman recorded fifty } \\
\text { score in PSL series. }\end{array}$ \\
\hline Sixes (6s) & Batsman made six runs in PSL series. \\
\hline Fours (4s) & $\begin{array}{l}\text { Boundaries in which batsman made four runs in } \\
\text { PSL series. }\end{array}$ \\
\hline
\end{tabular}

Table 2. Bowling attributes

\begin{tabular}{|l|l|}
\hline $\begin{array}{l}\text { Bowling } \\
\text { Features }\end{array}$ & Description \\
\hline Bowlers & $\begin{array}{l}\text { Name of the Bowlers who bowled in PSL } \\
\text { series. }\end{array}$ \\
\hline Wickets & Total wickets taken by bowlers in PSL series. \\
\hline Match & $\begin{array}{l}\text { Total matches bowled by bowlers in PSL } \\
\text { series. }\end{array}$ \\
\hline $\begin{array}{l}\text { Bowling } \\
\text { average (Ave) }\end{array}$ & $\begin{array}{l}\text { Average of total runs scored per wickets in } \\
\text { PSL series. } \\
\text { ( ave = runs / wkts ) }\end{array}$ \\
\hline $\begin{array}{l}\text { Bowlers } \\
\text { economy rate } \\
\text { (Econ) }\end{array}$ & $\begin{array}{l}\text { Average of total runs recorded per over } \\
\text { bowled in PSL series. }\end{array}$ \\
\hline $\begin{array}{l}\text { Bowlers strike } \\
\text { rate (SR) }\end{array}$ & $\begin{array}{l}\text { Average of total balls which is bowled per } \\
\text { wicket taken in PSL series . ( SR = } \\
\text { balls/wkts) }\end{array}$ \\
\hline $\begin{array}{l}\text { Catch outs } \\
\text { (Ct) }\end{array}$ & \begin{tabular}{l} 
Total catches taken by bowlers in PSL series. \\
\hline Overs
\end{tabular} $\begin{array}{l}\text { Total overs have been bowled by bowlers in } \\
\text { PSL series. }\end{array}$ \\
\hline
\end{tabular}

\subsection{Relative Feature Score Computation}

Estimate the individual value of each feature using correlation. With the help of correlation matrix this work identified how the factors are interrelated to target variable which is run score made by batsmen and wickets taken by bowlers from batting and bowling attributes respectively. The value of target variable may be maximize or minimize due to positive and negative correlation.

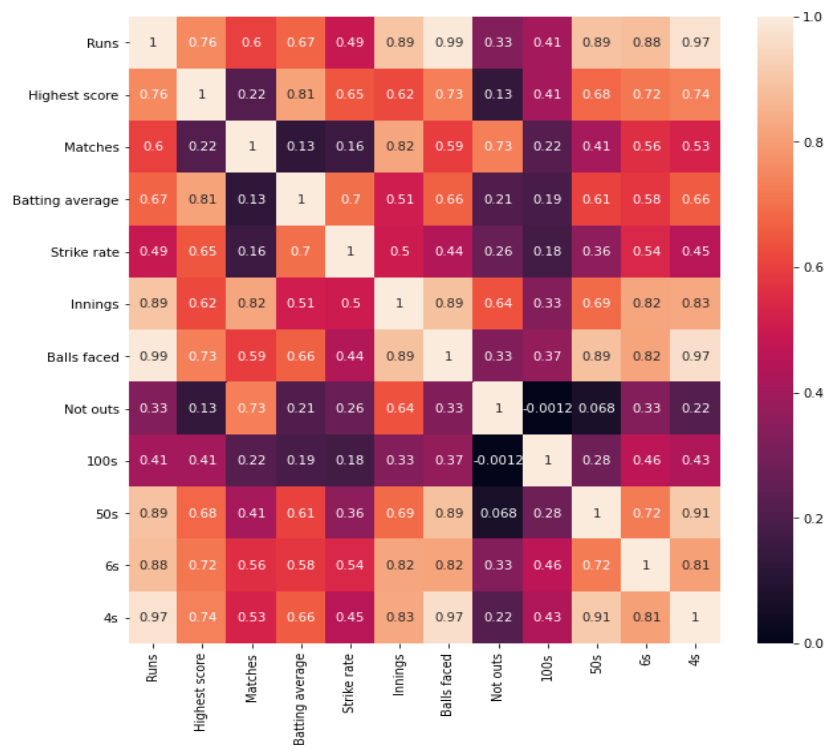

Fig 3: Correlation matrix of batting attributes

Figure 3 shows the correlation of batting features in which Runs score is target variable correlated to other factors as Balls faced is extremely correlated with Runs followed by $50 \mathrm{~s}$, innings, $6 \mathrm{~s}$, highest score, batting average and matches whereas not outs, strike rate and 100 s are slightly correlated with Runs. Finally feature score of batting attributes are calculated using following formula:

Feature score for batting attributes $\left(\mathrm{FS}_{1}\right)=$ (Runs*0.97+Highestscore*0.74+Matches*0.53+Battingave rage $* 0.66+$ Strikerate $* 0.45+$ Innings $* 0.83+$ Ballsfaced $* 0.97$ +Not outs*0.22+100s*0.43+50s*0.91+ $6 \mathrm{~s} * 0.81+4 \mathrm{~s} * 1)$

Where 0.97, 0.74, 0.53, 0.66, 0.45, 0.83, 0.97, 0.22, 0.43, $0.91,0.81$ and 1 are experimental variables used to rank the individual factors of top fifteen batsmen pronounced above in computation of feature score for batting attributes.

Feature score for bowling attributes $\left(\mathbf{F S}_{2}\right)=$ (Wickets*0.96+Match*0.89+Ave*(-0.038) +Econ*(-0.29) + $\mathrm{SR} * \mathbf{0 . 0 5 8 + C t} * \mathbf{0 . 6 6 + O v e r s} * \mathbf{1})$

Where $0.96,0.89,-0.038,-0.29,0.058,0.66$ and 1 are analytically observed variables to rank the top fifteen bowlers of PSL show in Figure 4.

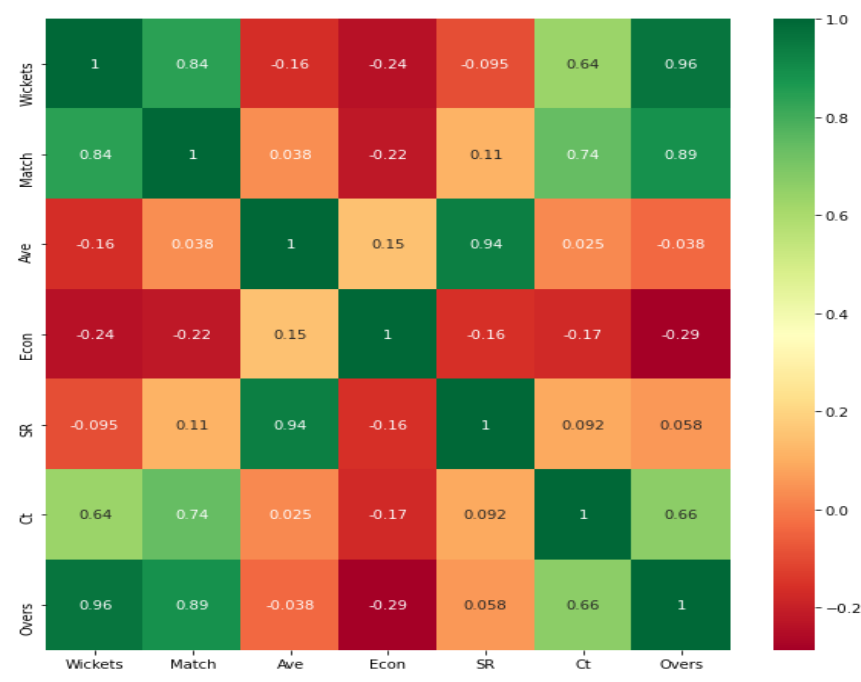

Fig 4: Correlation matrix for bowling attributes 


\section{RESULTS ANALYSIS \& DISCUSSION}

\subsection{Batting Analysis}

In first predictive model, top batsmen are estimated considering batting competencies of PSL players. In this model, thirteen batting attributes are used as batsmen name, runs made by batsmen, batsmen innings, batting average, sixes, fours, centuries, total matches, highest score of batsmen, balls faced by batsmen, not outs, strike rate and fifties made by batsmen which have been exclusively normalized using correlation matrix. For implementation, model is trained and Scikit library is used for ML algorithms. Predictive model individually examines the proficiencies of batsmen and then fused all batting capabilities together. For batsmen analysis, group the batsmen data by batsmen names and merge all their batting aptitudes organized. After that sort the calculated values in decreasing order and ranking top to bottom best fifteen batsmen of PSL tournament based on feature score shows in Figure 5. Babar Azam and Kamran Akmal have best feature score of 3102.67 and 3033.88 respectively.

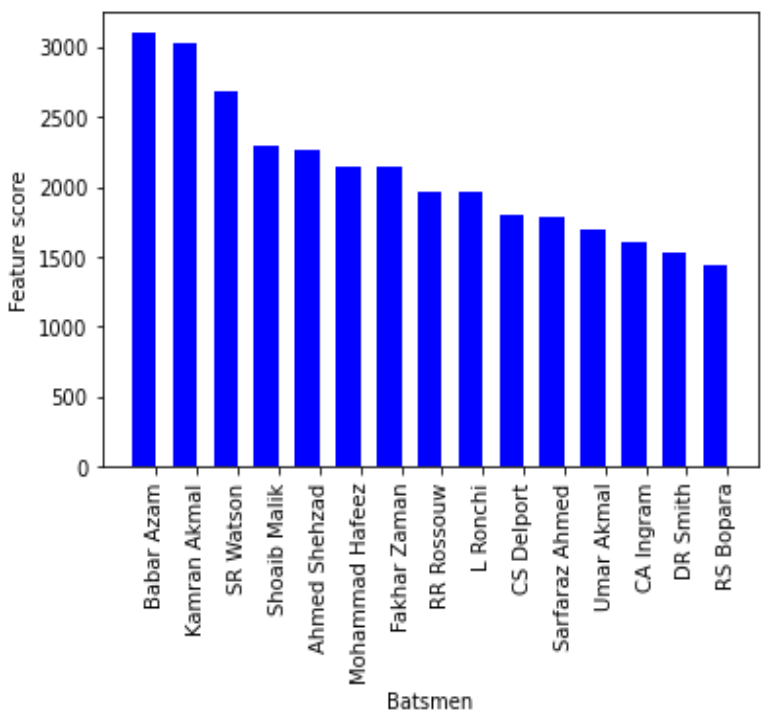

Fig 5: Top Batsmen of PSL based on Feature score

Figure 6 shows the ranking of top fifteen batsmen of PSL whereas Figure 7 indicates a whole list of top 119 PSL batsmen ranked who played at least eight matches in PSL season (2016-2020).

Batting-Index $=($ Batting-Average $) *($ Strike-rate $) / 100$

Batsmen performance also depends on highest value of batting index shows in Figure 8.

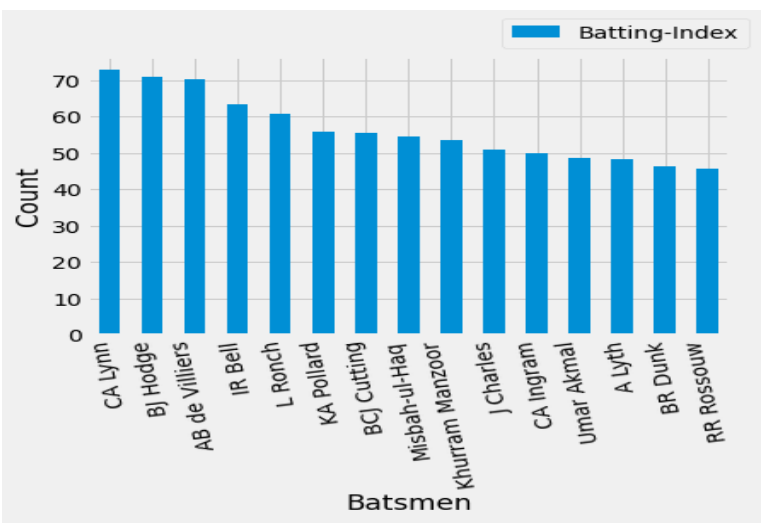

Fig 8: Batting index of PSL players

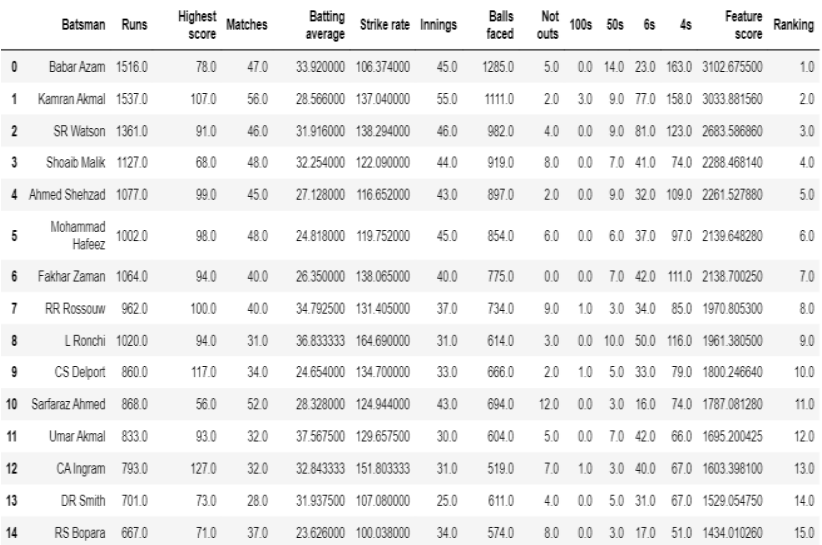

Fig 6: Top fifteen batsmen in PSL

\begin{tabular}{|c|c|c|c|c|c|}
\hline & Runs & $\begin{array}{c}\text { Highest } \\
\text { score }\end{array}$ & Matches & $\begin{array}{l}\text { Batting } \\
\text { average }\end{array}$ & $\begin{array}{l}\text { Strike } \\
\text { rate }\end{array}$ \\
\hline count & 119.000000 & 119.000000 & 119.000000 & 119.000000 & 119.000000 \\
\hline mean & 322.941176 & 50.000000 & 23.201681 & 19.037301 & 108.836283 \\
\hline std & 333.654248 & 30.629553 & 13.461546 & 11.124896 & 34.129366 \\
\hline min & 1.000000 & 1.000000 & 8.000000 & 0.000000 & 0.000000 \\
\hline $25 \%$ & 76.000000 & 23.500000 & 12.000000 & 10.614584 & 95.235000 \\
\hline $50 \%$ & 221.000000 & 50.000000 & 19.000000 & 18.265000 & 115.817500 \\
\hline $75 \%$ & 448.500000 & 73.000000 & 32.000000 & 27.861666 & 130.127500 \\
\hline $\max$ & 1537.000000 & 127.000000 & 56.000000 & 50.192500 & 179.740000 \\
\hline & Innings & $\begin{array}{l}\text { Balls } \\
\text { faced }\end{array}$ & $\begin{array}{l}\text { Not } \\
\text { outs }\end{array}$ & $100 \mathrm{~s}$ & $50 \mathrm{~s}$ \\
\hline count & 119.000000 & 119.000000 & 119.000000 & 119.000000 & 119.000000 \\
\hline mean & 17.016807 & 252.655462 & 3.394958 & 0.067227 & 1.638655 \\
\hline std & 12.115953 & 254.731711 & 3.380448 & 0.337767 & 2.516828 \\
\hline min & 2.000000 & 2.000000 & 0.000000 & 0.000000 & 0.000000 \\
\hline $25 \%$ & 8.000000 & 58.500000 & 1.000000 & 0.000000 & 0.000000 \\
\hline $50 \%$ & 13.000000 & 177.000000 & 2.000000 & 0.000000 & 1.000000 \\
\hline $75 \%$ & 23.000000 & 356.500000 & 5.000000 & 0.000000 & 2.000000 \\
\hline $\max$ & 55.000000 & 1285.000000 & 14.000000 & 3.000000 & 14.000000 \\
\hline & $6 \mathrm{~s}$ & $4 \mathrm{~s}$ & Ranking & & \\
\hline count & 119.000000 & 119.000000 & 119.000000 & & \\
\hline mean & 13.226891 & 28.184874 & 60.000000 & & \\
\hline std & 15.382930 & 32.458309 & 34.496377 & & \\
\hline min & 0.000000 & 0.000000 & 1.000000 & & \\
\hline $25 \%$ & 2.000000 & 5.000000 & 30.500000 & & \\
\hline $50 \%$ & 8.000000 & 17.000000 & 60.000000 & & \\
\hline $75 \%$ & 17.000000 & 40.000000 & 89.500000 & & \\
\hline $\max$ & 81.000000 & 163.000000 & 119.000000 & & \\
\hline
\end{tabular}

Fig 7: Descriptive analysis of top batsmen in PSL

In this analysis various sizes of training and testing sets are performed to idealize the particular percentage of given data. Five machine learning approaches are applied as SVM, Naive Bayes, Random Forest, KNN and Linear Regression to estimate batting performance of top batsmen in PSL series with best combination of training and testing model accuracy. The final results of dualistic percentage are given in Table 3 which shows the accuracies estimated by different approaches to predict the Runs score.

Table 3. Runs prediction with different sizes of training and testing set

\begin{tabular}{|c|c|c|c|c|c|}
\hline \multirow[b]{2}{*}{ Classifier } & \multicolumn{5}{|c|}{ Accuracy (\%) } \\
\hline & $\begin{array}{l}85 \% \\
\text { train } \\
\& \\
15 \% \\
\text { test }\end{array}$ & $\begin{array}{l}80 \% \\
\text { train } \\
\& \\
20 \% \\
\text { test }\end{array}$ & $\begin{array}{l}70 \% \\
\text { train } \\
\text { \& } \\
30 \% \text { te } \\
\text { st }\end{array}$ & $\begin{array}{l}\mathbf{6 5 \%} \\
\text { train } \\
\text { \& } \\
\mathbf{3 5 \%} \\
\text { test }\end{array}$ & $\begin{array}{l}60 \% \\
\text { train } \\
\& \\
40 \% \\
\text { test }\end{array}$ \\
\hline $\begin{array}{l}\text { Random } \\
\text { Forest } \\
\end{array}$ & 100 & 100 & 100 & 99.89 & 99.52 \\
\hline SVM & 94.00 & 93.24 & 90.03 & 89.68 & 87.57 \\
\hline Naive Bayes & 73.02 & 69.24 & 66.40 & 64.24 & 64.04 \\
\hline $\begin{array}{l}\text { Linear } \\
\text { Regression }\end{array}$ & 80.56 & 86.32 & 80.56 & 82.90 & 81.98 \\
\hline KNN & 76.23 & 84.45 & 86.00 & 77.8 & 89.45 \\
\hline
\end{tabular}


It is apparent from Table 3 that random forest gives best prediction results for batting feature in all training and testing sets. Accuracies of Random forest, SVM and Naive Bayes increase as size of training set is increased except linear regression and $\mathrm{KNN}$. In this case prediction accuracies increase when the size of training dataset is decreased. In prediction of Runs score the random forest gives an accuracy of $100 \%$ when $85 \%, 80 \%$ and $70 \%$ of data is used as training set whileat $65 \%$ and $60 \%$ of training data this model providesaccuracies of $99.89 \%$ and $99.52 \%$ respectively. For predicting the runs, SVM provides highest accuracy of $94 \%$ when training data is $85 \%$ used whereas it gives low accuracy of $87.57 \%$ when training model used as $60 \%$. Naive Bayes estimate runs score with lowest accuracy of $64.04 \%$ when training data is used $60 \%$ and predicts the Runs with highest accuracy of $73.02 \%$ at $85 \%$ of training set. Linear regression evaluated the runs score with minimum accuracy of $80.56 \%$ when training set is used as $70 \%$ and $85 \%$ respectively and at $80 \%$ of training data this model estimated the maximum accuracy of $86.32 \%$ to predict the Runs score. KNN predicts the runs score with high accuracy of 89.45 when $60 \%$ training data is used and predicts the runs with low accuracy of $76.23 \%$ at $85 \%$ of training set. The top five batting players of PSL are acknowledged here as Babar Azam, Kamran Akmal, SR Watson, Shoaib Malik and Ahmed Shehzad who made maximum runs of 1516, 1537, 1361, 1127 and 1077 respectively. Figure 9 indicates the plot of batting performance of top batsmen prediction in term of accuracy metrics when training set is $85 \%$.

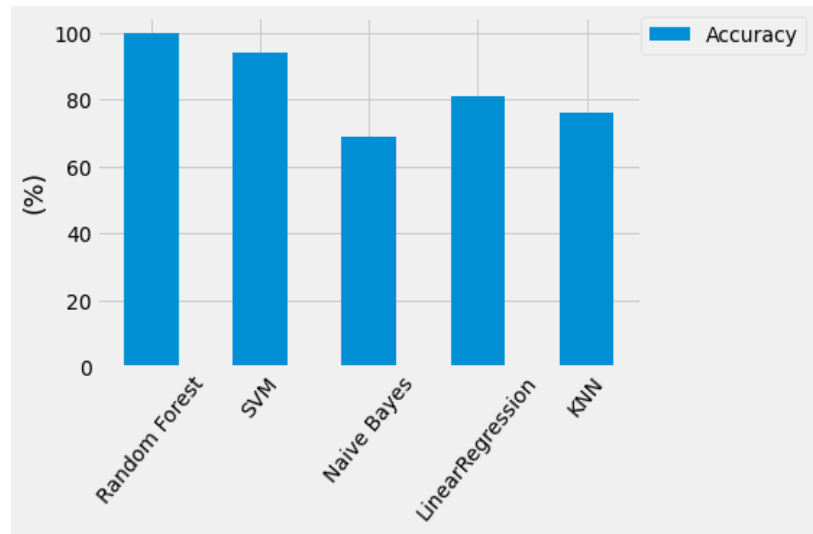

Fig 9: Accuracy metrics evaluated using machine learning algorithms for runs prediction of top batsmen in PSL using $85 \%$ of data as training

Estimating precision, recall and f1-score results for Runs (total number of runs made by top batsmen) are shown in Figure 10 compatible with accuracy when $85 \%$ of data is used as training model. Random Forest approach outstripped as compared to other techniques with the precision, recall and $\mathrm{f1-}$ score of $100 \%$ for Runs prediction owing to null character false positives (FPs) and false negatives (FNs). This model is capable to properly classify all samples rendering to confusion matrix while SVM, Naive Bayes, KNN and Linear regression incorrectly classified 7, 25, 17 and 32 instances respectively for predicting the Runs. These wrongly predicted samples enlarged the FPs and FNs and eventually gave lower results for precision, recall and f1-score. These performance metrics are acquired by SVM is $89 \%, 94 \%$ and $91 \%$ as precision, recall and f1 score respectively and Naive Bayes gave $84 \%$, $86 \%$ and $90 \%$ respectively. Linear regression provides $83 \%$ precision, $87 \%$ recall and $90 \%$ f1-score while $\mathrm{KNN}$ gives $86 \%$ precision, $90 \%$ recall and $92 \%$ f1-score for runs prediction.

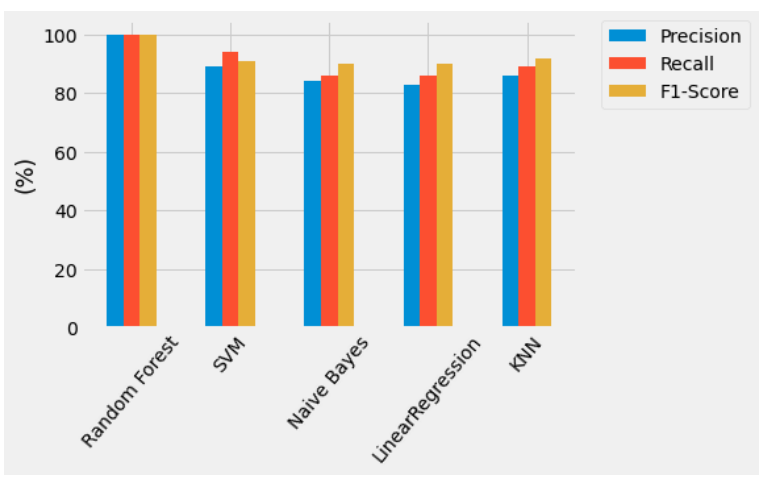

Fig 10: Precision, recall and f1_score evaluated using machine learning algorithms for top batsmen in PSL using $\mathbf{8 5 \%}$ of data as training set

\subsection{Bowling Analysis}

In second predictive model, eight bowling features have been used as response to scrutinize the top fifteen bowlers in PSL using feature score formula defined in section 3 . The factors used during analysis are: bowlers, wickets taken by bowler's name, matches played by bowlers, bowling average, bowler's innings, bowler's economy rate, bowling strike rate, total catches, and total overs bowled by bowlers in PSL series. Top fifteen batsmen based on feature score shows in Figure 11. WahabRiaz and Mohammad Irfan have top feature score of 333.66 and 322.3 respectively.

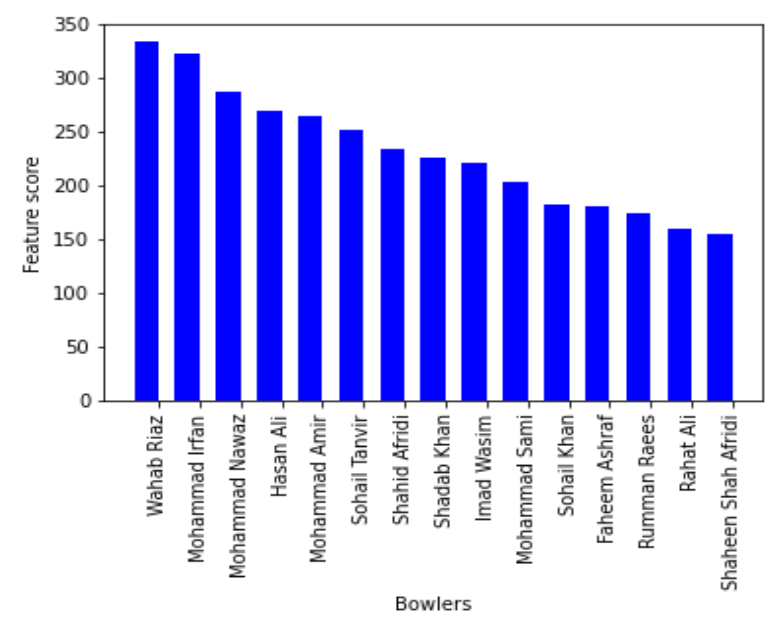

Fig 11: Top bowlers based on feature score

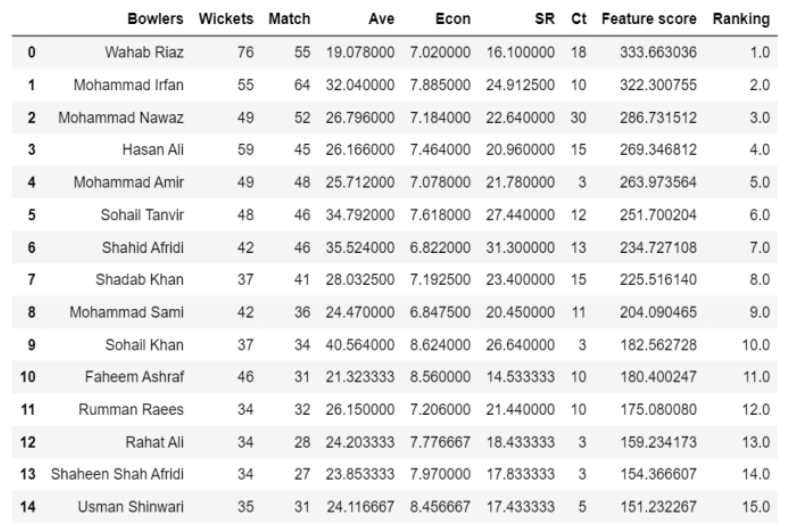

Fig 12: Top fifteen bowlers in PSL 


\begin{tabular}{|c|c|c|c|c|c|}
\hline & Wickets & Match & Ave & Econ & SR \ \\
\hline count & 98.000000 & 98.000000 & 98.000000 & 98.000000 & 98.000000 \\
\hline mean & 16.265306 & 17.765306 & 29.964723 & 8.101371 & 22.269328 \\
\hline std & 14.978026 & 14.297863 & 10.724092 & 1.103296 & 8.035061 \\
\hline $\min$ & 1.000000 & 2.000000 & 10.600000 & 5.373333 & 9.600000 \\
\hline $25 \%$ & 5.000000 & 6.250000 & 22.637500 & 7.362500 & 16.693750 \\
\hline $50 \%$ & 10.500000 & 14.000000 & 27.595000 & 7.935000 & 20.980000 \\
\hline $75 \%$ & 23.000000 & 27.000000 & 35.023000 & 8.623000 & 25.525000 \\
\hline $\max$ & 76.000000 & 62.000000 & 71.000000 & 11.500000 & 54.000000 \\
\hline & $\mathrm{Ct}$ & Overs & Ranking & & \\
\hline count & 98.000000 & 98.000000 & 98.000000 & & \\
\hline mean & 4.908163 & 52.046939 & 49.500000 & & \\
\hline std & 5.197316 & 48.031242 & 28.434134 & & \\
\hline $\min$ & 0.000000 & 8.000000 & 1.000000 & & \\
\hline $25 \%$ & 1.000000 & 16.000000 & 25.250000 & & \\
\hline $50 \%$ & 3.000000 & 33.200000 & 49.500000 & & \\
\hline $75 \%$ & 7.000000 & 70.500000 & 73.750000 & & \\
\hline $\max$ & 30.000000 & 208.000000 & 98.000000 & & \\
\hline
\end{tabular}

Fig 13: Descriptive analysis of top bowlers in PSL

Figure 12 indicates top fifteen bowlers based on their bowling capabilities in PSL tournament while Figure 13 shows complete ranking list of top 98 PSL bowlers who bowled in at least eight overs in PSL season (2016-2020). Both Figures also indicate the best bowling performance based on thoroughgoing taking outs by bowlers from batsmen, minimum number of average, strike rate and economy rate as well.

\section{Bowling-Index $=($ Bowling-Average $) *($ Bowling-Strike} rate) $/ \mathbf{1 0 0}$

A best bowling performance depends on lowest value of bowling index shows in Figure 14.

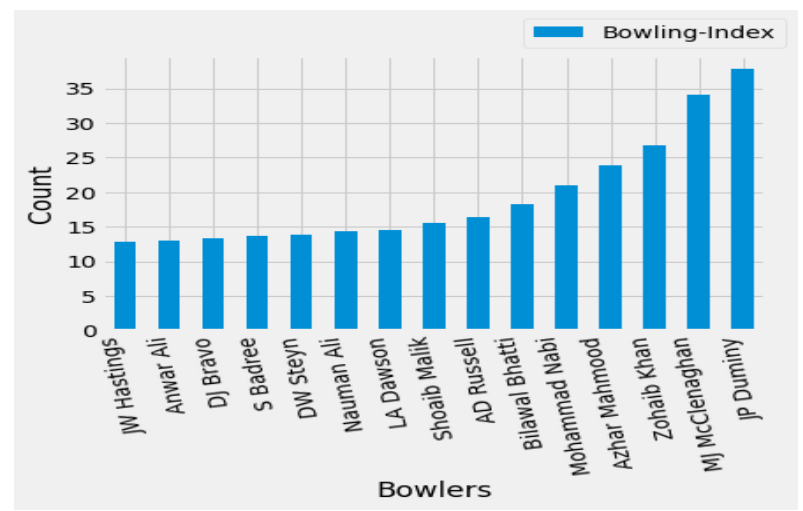

Fig 14: Bowling index of PSL players

In this examination several train's and test's sizes are used to predict the wickets taken by top bowlers. Table 4 shows the different sizes of train and test set to choose the best model accuracy applying five machine learning techniques to predict the wickets.

Table 4. Wickets Prediction with different sizes of training and testing sets

\begin{tabular}{|c|c|c|c|c|c|}
\hline \multirow[b]{2}{*}{ Classifier } & \multicolumn{5}{|c|}{ Accuracy (\%) } \\
\hline & $\begin{array}{l}85 \% \\
\text { train } \\
\& \\
15 \% \\
\text { test }\end{array}$ & $\begin{array}{l}80 \% \\
\text { train } \\
\& \\
20 \% \\
\text { test }\end{array}$ & $\begin{array}{l}70 \% \\
\text { train } \\
\& \\
30 \% \text { te } \\
\text { st }\end{array}$ & $\begin{array}{l}\mathbf{6 5 \%} \\
\text { train } \\
\mathbf{\&} \\
\mathbf{3 5 \%} \\
\text { test }\end{array}$ & $\begin{array}{l}60 \% \\
\text { train } \\
\& \\
40 \% \\
\text { test }\end{array}$ \\
\hline $\begin{array}{l}\text { Random } \\
\text { Forest }\end{array}$ & 100 & 100 & 100 & 98.54 & 99.85 \\
\hline SVM & 89.00 & 73.44 & 70.29 & 68.46 & 66.23 \\
\hline $\begin{array}{l}\text { Naive } \\
\text { Bayes }\end{array}$ & 47.74 & 49.15 & 47.30 & 53.55 & 58.50 \\
\hline $\begin{array}{l}\text { Linear } \\
\text { Regression }\end{array}$ & 67.09 & 48.98 & 47.00 & 47.97 & 39.68 \\
\hline KNN & 41.56 & 45.78 & 45.31 & 48.99 & 44.34 \\
\hline
\end{tabular}

From Table 4 training and testing models are analyzed that random forest is best approach to predict the wickets from bowling attributes with high accuracy of $100 \%$ when $85 \%$, $80 \%$ and $70 \%$ of data applied as train the model. Random forest gives lowest accuracy of $98.54 \%$ at $65 \%$ of training model. Accuracies of random forest, SVM and linear regression maximize as size of training model is maximized except in case of KNN and Naive Bayes. Accuracies of Naive Bayes and KNN increase as size of training model is decreased. SVM and linear regression provide the high accuracies of $89 \%$ and $67.09 \%$ respectively when $85 \%$ used as training set for the prediction of wickets and these both model provide low accuracies of $66.23 \%$ and $39.68 \%$ respectively when $60 \%$ training model is used. Naive Bayes gives lowest accuracy of $47.3 \%$ at $70 \%$ train set. Naive Bayes provides high accuracy of $58.50 \%$ at $60 \%$ of training set while $\mathrm{KNN}$ gives low accuracy of $41.56 \%$ when $85 \%$ of data is used as train model. KNN predicts the wickets with high accuracy of $48.99 \%$ when training model is used as $65 \%$. Figure 15 indicates the plot of bowling features of top bowler's estimation in term of accuracy metrics when $85 \%$ of data used as train model.

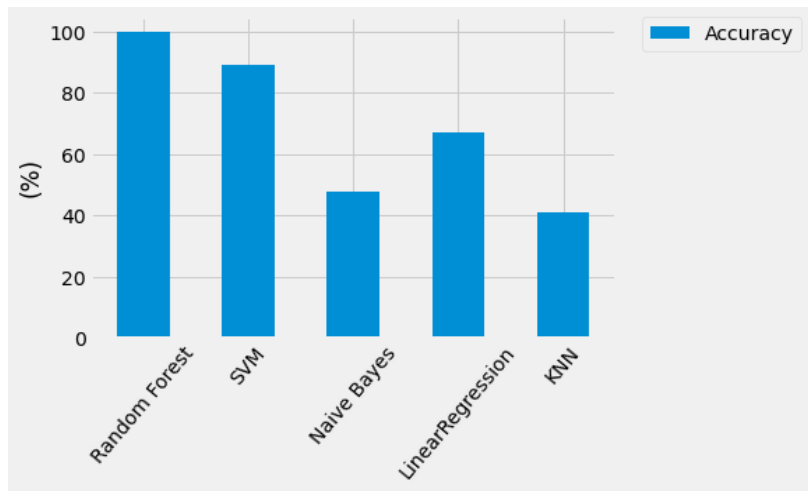

Fig 15:Accuracy metrics estimated using machine learning algorithms for wickets prediction of top bowlers in PSL

Precision, recall and f1 score results are evaluated for scrutinizing the "Wickets" prediction against the top batsmen feature when $85 \%$ of data is used as train model which shown in Figure 16 attuned with accuracy. In this, Random Forest approach outshined like first predictive model by means of all other techniques with the precision, recall and f1 score metrics of $100 \%$ as a result of null character false positives (FPs) and false negatives (FNs). This model proficient to correctly classify all samples interpreting to confusion matrix while SVM, Naive Bayes, Linear regression and KNN misclassified 32, 42, 9 and 17 samples respectively. These wrongly predicted instances achieve higher FPs and FNs values which ultimately decreasing the precision, recall and f1 score performance metrics. SVM approach gave precision, recall and $\mathrm{f1}$ score results about $56 \%, 82 \%$ and $67 \%$ respectively and Naive Bayes provides the results of $36 \%, 73 \%$ and $48 \%$ respectively due to higher FPs and FNs samples for prediction of wickets at $85 \%$ train set. Linear regression predicts the wickets with $67 \%$ of precision, $96 \%$ of recall and $80 \%$ of f1score whereas KNN provides precision, recall and $\mathrm{f1}$-score of $44 \%, 94 \%$ and $64 \%$ respectively. It means, naive Bayes approach was incapable to increase the predictive results via all other techniques exposed an undesirable outcomes regarding to precision, recall and $\mathrm{f} 1$ score. Top five bowling players of PSL are predicted as WahabRiaz, Hassan Ali, Mohammad Irfan, Mohammad Amir and Mohammad Nawaz who has taken 76, 59, 55, 49 and 48 wickets respectively from batsmen. 


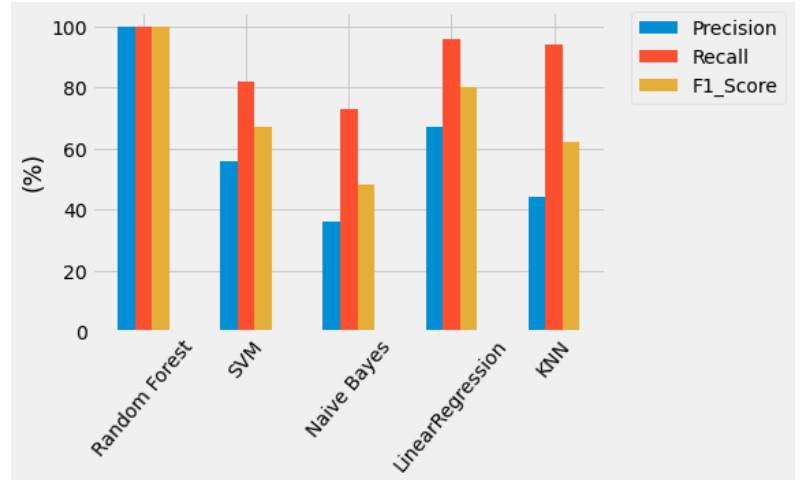

Fig 16: Precision, recall and f1_score evaluated using machine learning algorithms for top bowlers in PSL

\section{CONCLUSION \& FUTURE WORK}

This study takes an imperious step to select the best players for PSL matches assuming a huge part in team's triumph and used previous five years of PSL data to predict the match outcome. Machine learning techniques are applied to estimate the final predictions by ranking the top players of PSL by dint of batting and bowling capabilities regarding accuracy, precision, recall and f1-score. In this research, set of 119 batsmen and 98 bowlers has been examined who played and bowled in at least eight matches and eight overs of PSL respectively. Four distinct machine learning classification techniques for scrutinizing the final outcomes have been executed and compared. First predictive model gave marginally high performance than second predictive model with evaluation parameters. Random forest performed better for both models as compared to other algorithms with an accuracy of $100 \%$ for analyzing runs made by batsmen and wickets taken by bowlers respectively. SVM gave equally high results concerning accuracy for predicting runs by batsmen and wickets taken by bowlers which are $94 \%$ and $89 \%$ respectively where as Naive Bayes gave low accuracy of $69 \%$ for runs prediction and $48 \%$ for wickets prediction. Linear regression predicted the runs with accuracy of $80.56 \%$ and predicted the wickets with accuracy of $67.09 \%$ while KNN predicted the runs and wickets with accuracy of 76.23 and $41.56 \%$ respectively. Random Forest classifier outperformed with the precision, recall and f1-score of $100 \%$ by means of zero result value of FPs and FNs for prediction of both runs made by batsmen and wickets taken by bowlers. It is concluded that random forest and SVM gave approximately equal high result for batting feature in accuracy metrics whereas Naive Bayes, Linear Regression and KNN gave low results which are not much satisfactory.Ranking of top fifteen batsmen and bowlers are depicted in Figure 6 and Figure 12 respectively. An exact prediction of top players' selection in match series will help the team managers and researchers involved in Cricket field to select the best players for team winning chances. These comparable models can also be worked for other formats of cricket like Test matches and ODI tournaments. In future, deep learning classification approach will be used in order to apprehend more valuable factors that can conceivably increase the prediction accuracy.

\section{REFERENCES}

[1] Jayalath, K. P. 2018. A machine learning approach to analyze ODI cricket predictors. Journal of Sports Analytics, 4(1), 73-84.

[2] Tekade, P., Markad, K., Amage, A., \&Natekar, B. 2020. Cricket match outcome prediction using machine learning. International journal, 5(7).

[3] Kapadia, K., Abdel-Jaber, H., Thabtah, F., \&Hadi, W. 2020. Sport analytics for cricket game results using machine learning: An experimental study. Applied Computing and Informatics.

[4] Munir, F., Hasan, M., \& Ahmed, S. 2015. Predicting a T20 cricket match result while the match is in progress (Doctoral dissertation, Brac University).

[5] Kampakis, S., \& Thomas, W. 2015. Using machine learning to predict the outcome of english county twenty over cricket matches. arXiv preprint arXiv:1511.05837.

[6] Ahmed, W., Amjad, M., Junejo, K., Mahmood, T., \& Khan, A. 2020. Is the performance of a cricket team really unpredictable? a case study on pakistan team using machine learning. Indian Journal of Science and Technology, 13(34), 3586-3599.

[7] Shetty, M., Rane, S., Pandita, C., \&Salvi, S. 2020. Machine learning-based Selection of Optimal sports Team based on the Players Performance. In 2020 5th International Conference on Communication and Electronics Systems (ICCES) (pp. 1267-1272). IEEE.

[8] Singh, S., Aggarwal, Y., \&Kundu, K. 2020. Quantitative Analysis of Forthcoming ICC Men's T20 World Cup 2020 Winner Prediction using Machine Learning. International Journal of Computer Applications, 975, 8887.

[9] Nimmagadda, A., Kalyan, N. V., Venkatesh, M.,Teja, N. N. S., \&Raju, C. G. 2018. Cricket score and winning prediction using data mining. International Journal for Advance Research and Development, 3(3), 299-302.

[10] Pathak, N., \&Wadhwa, H. 2016. Applications of modern classification techniques to predict the outcome of ODI cricket. Procedia Computer Science, 87, 55-60.

[11] Jhanwar, M. G., \&Pudi, V. 2016. Predicting the Outcome of ODI Cricket Matches: A Team Composition Based Approach. In MLSA@ PKDD/ECML.

[12] Kumar, J., Kumar, R., \& Kumar, P. 2018. Outcome prediction of ODI cricket matches using decision trees and MLP networks. In 2018 First International Conference on Secure Cyber Computing and Communication (ICSCCC) (pp. 343-347). IEEE.

[13] Somaskandhan, P., Wijesinghe, G., Wijegunawardana, L. B., Bandaranayake, A., \&Deegalla, S. 2017, December. Identifying the optimal set of attributes that impose high impact on the end results of a cricket match using machine learning. In 2017 IEEE International Conference on Industrial and Information Systems (ICIIS) (pp. 1-6). IEEE.

[14] Thenmozhi, D., Mirunalini, P., Jaisakthi, S. M., Vasudevan, S., Kannan, V. V., \&Sadiq, S. 2019. MoneyBall-Data Mining on Cricket Dataset. In 2019 International Conference on Computational Intelligence in Data Science (ICCIDS) (pp. 1-5). IEEE.

[15] [Online]. Available: https://www.espncricinfo.com.

[16] İshrat, R. İ. A. Z., Mushtaq, N., Jillani, M. M., \&Navaz, U. 2019. Performance analysis of Pakistan super league players using principle component analysis approach. Scientific Journal of Mehmet AkifErsoy University, 2(4), 127-135. 